\title{
Dynamics of a modified Leslie-Gower predator-prey model with Holling-type II schemes and a prey refuge
}

\author{
Qin Yue*
}

*Correspondence:
xhper1978@sina.com
College of Finance
and Mathematics,
West Anhui University,
Liuan 237000, Anhui, People's
Republic of China

${ }^{*}$ Correspondence: xhper1978@sina.com and Mathematics, West Anhui University, Republic of China

\begin{abstract}
We propose a modified Leslie-Gower predator-prey model with Holling-type II schemes and a prey refuge. The structure of equilibria and their linearized stability is investigated. By using the iterative technique and further precise analysis, sufficient conditions on the global attractivity of a positive equilibrium are obtained. Our results not only supplement but also improve some existing ones. Numerical simulations show the feasibility of our results.
\end{abstract}

Keywords: Leslie-Gower, Equilibrium, Global attractivity, Iterative, Refuge

\section{Background}

The dynamic relationship between predators and their preys has long been and will continue to be one of the dominant themes in both ecology and mathematical ecology due to its universal existence and importance. Leslie $(1948,1958)$ introduced the following two species Leslie-Gower predator-prey model:

$$
\left\{\begin{array}{l}
\dot{x}(t)=\left(r_{1}-b_{1} x\right) x-p(x) y, \\
\dot{y}(t)=\left(r_{2}-\frac{a_{2} y}{x}\right) y
\end{array}\right.
$$

where $x(t), y(t)$ stand for the population (the density) of the prey and the predator at time $t$, respectively. The parameters $r_{1}$ and $r_{2}$ are the intrinsic growth rates of the prey and the predator, respectively. $b_{1}$ measures the strength of competition among individuals of species $x$. The value $\frac{r_{1}}{b_{1}}$ is the carrying capacity of the prey in the absence of predation. The predator consumes the prey according to the functional response $p(x)$ and grows logistically with growth rate $r_{2}$ and carrying capacity $\frac{r_{2} x}{a_{2}}$ proportional to the population size of the prey (or prey abundance). The parameter $a_{2}$ is a measure of the food quantity that the prey provides and converted to predator birth. The term $y / x$ is the Leslie-Gower term which measures the loss in the predator population due to rarity (per capita $y / x$ ) of its favorite food. Leslie model is a predator-prey model where the carrying capacity of the predator is proportional to the number of prey, stressing the fact that there are upper limits to the rates of increase in both prey $x$ and predator $y$, which are not recognized in the Lotka-Volterra model.

(c) 2016 Yue. This article is distributed under the terms of the Creative Commons Attribution 4.0 International License (http://creativecommons.org/licenses/by/4.0/), which permits unrestricted use, distribution, and reproduction in any medium, provided you give appropriate credit to the original author(s) and the source, provide a link to the Creative Commons license, and indicate if changes were made. 
As was pointed out by Aziz-Alaoui and Daher (2003), in the case of severe scarcity, $y$ can switch over to other populations but its growth will be limited by the fact that its most favorite food $x$ is not available in abundance. In order to solve such deficiency in system (1), Aziz-Alaoui and Daher (2003) proposed and studied the following predatorprey model with modified Leslie-Gower and Holling-type II schemes:

$$
\left\{\begin{array}{l}
\dot{x}(t)=\left(r_{1}-b_{1} x-\frac{a_{1} y}{x+k_{1}}\right) x, \\
\dot{y}(t)=\left(r_{2}-\frac{a_{2} y}{x+k_{2}}\right) y,
\end{array}\right.
$$

where $r_{1}, b_{1}, r_{2}, a_{2}$ have the samemeaning as in system (1). $a_{1}$ is the maximum value which per capita reduction rate of $x$ can attain; $k_{1}$ and $k_{2}$ measure the extent to which environment provides protection to prey $x$ and to predator $y$ respectively. They obtained the boundedness and global stability of positive equilibrium of system (1). Since then, many scholars considered system (2) and its non-autonomous versions by incorporating delay, impulses, harvesting, stochastic perturbation and so on (see, for example, Yu 2012; Nindjin et al. 2006; Yafia et al. 2007, 2008; Nindjin and Aziz-Alaoui 2008; Gakkhar and Singh 2006; Guo and Song 2008; Song and Li 2008; Zhu and Wang 2011; Liu and Wang 2013; Kar and Ghorai 2011; Huo et al. 2011; Li et al. 2012; Liu et al. 2013; Gupta and Chandra 2013; Ji et al. 2009, 2011; Yu 2014; Yu and Chen 2014; Yue 2015). In particular, $\mathrm{Yu}(2012)$ studied the structure, linearized stability and the global asymptotic stability of equilibria of (2) and obtained the following result (see Theorem 3.1 in Yu 2012):

Theorem 1 Assume that

$$
\begin{array}{ll}
a_{1} r_{1} r_{2}+a_{1} b_{1} r_{2} k_{2}<a_{2} b_{1} r_{1} k_{1}, & \left(C_{1}\right) \\
2 a_{2} b_{1} M+\left(a_{2} b_{1} k_{1}-a_{2} r_{1}-a_{1} r_{2}\right)>0, & \left(C_{2}\right)
\end{array}
$$

hold, where $M=\frac{r_{1} k_{1}-a_{1} L}{b_{1} k_{1}}$ and $L=\frac{r_{1} r_{2}+b_{1} r_{2} k_{2}}{a_{2} b_{1}}$, then system (2) has a unique positive equilibrium which is globally attractive.

As was pointed out by Kar (2005), mite predator-prey interactions often exhibit spatial refugia which afford the prey some degree of protection from predation and reduce the chance of extinction due to predation. In Kar (2005), Tapan Kumar Kar had considered a predator-prey model with Holling type II response function and a prey refuge. The author obtained conditions on persistent criteria and stability of the equilibria and limit cycle for the system. For more works on this direction, one could refer to Kar (2005), Srinivasu and Gayatri (2005), Ko and Ryu (2006), Huang et al. (2006), Kar (2006), González-Olivares and Ramos-Jiliberto (2003), Ma et al. (2009), Chen et al. (2009, 2010, 2012), Ji and Wu (2010), Tao et al. (2011) and the references cited therein.

Although many authors have considered the dynamic behaviors of the modified Leslie-Gower model (Yu 2012; Nindjin et al. 2006; Yafia et al. 2007, 2008; Nindjin and AzizAlaoui 2008; Gakkhar and Singh 2006; Guo and Song 2008; Song and Li 2008; Zhu and Wang 2011; Liu and Wang 2013; Kar and Ghorai 2011; Huo et al. 2011; Li et al. 2012; Liu et al. 2013; Gupta and Chandra 2013; Ji et al. 2009, 2011; Yu 2014; Yu and Chen 2014; Yue 2015) and predator-prey with a prey refuge (Kar 2005; Srinivasu and Gayatri 2005; 
Ko and Ryu 2006; Huang et al. 2006; Kar 2006; González-Olivares and Ramos-Jiliberto 2003; Ma et al. 2009; Chen et al. 2009, 2010, 2012; Ji and Wu 2010; Tao et al. 2011), as far as we know, there are almost no literatures discussing the modified Leslie-Gower model with a prey refuge. Stimulated by the works of Kar (2005), Srinivasu and Gayatri (2005), Ko and Ryu (2006), Huang et al. (2006), Kar (2006), González-Olivares and Ramos-Jiliberto (2003), Ma et al. (2009), Chen et al. (2009, 2010, 2012), Ji and Wu (2010), Tao et al. (2011), we will extend model (2) by incorporating a refuge protecting $m x$ of the prey, where $m \in[0,1)$ is constant. This leaves $(1-m) x$ of the prey available to the predator, and modifying system (2) accordingly to the system:

$$
\left\{\begin{array}{l}
\dot{x}(t)=\left(r_{1}-b_{1} x-\frac{a_{1}(1-m) y}{(1-m) x+k_{1}}\right) x, \\
\dot{y}(t)=\left(r_{2}-\frac{a_{2} y}{(1-m) x+k_{2}}\right) y .
\end{array}\right.
$$

system (2) is the special case of (3) with $m=0$, i.e. there is no prey refuge. By using iterative technique and further precise analysis, we finally obtain the following main results:

Theorem 2 Suppose that

$$
a_{1}(1-m)^{2} r_{1} r_{2}+a_{1}(1-m) b_{1} r_{2} k_{2}<a_{2} b_{1} r_{1} k_{1}, \quad\left(C_{3}\right)
$$

holds, then system (3) has a unique positive equilibrium $\left(x^{*}, y^{*}\right)$ which is globally attractive.

Theorem 2 shows that $\lim _{t \rightarrow \infty} x(t)=x^{*}, \lim _{t \rightarrow \infty} y(t)=y^{*}$. Notice that $x^{*}$ and $y^{*}$ are only dependent with the coefficients of system (3), and independent of the solution of system (3). Thus we can get the following result:

Corollary 1 Suppose that $C_{3}$ holds, then system (2) is permanent.

When $m=0$ that is there is no prey refuge, (3) becomes to (2) and $C_{3}$ becomes to $C_{1}$, so as a direct corollary of Theorem 2 , we have:

Corollary 2 Suppose that $C_{1}$ holds, then system (2) has a unique positive equilibrium which is globally attractive.

Comparing with Theorem 1 , it follows from Corollary 2 that $C_{2}$ is superfluous, so our results improve the main results in $\mathrm{Yu}$ (2012). Moreover, when consider the case of no alternate prey, so $k_{2}=0$ (this is often called the Holling-Tanner model), by the similar proof of Theorem 2, we can obtain:

Corollary 3 Suppose that

$$
a_{1}(1-m)^{2} r_{1} r_{2}<a_{2} b_{1} r_{1} k_{1}, \quad\left(C_{4}\right)
$$

holds, then system (3) with $k_{2}=0$ has a unique positive equilibrium $\left(x^{*}, y^{*}\right)$ which is globally attractive. 
The remaining part of this paper is organized as follows. In section "Nonnegative equilibria and their linearized stability", we discuss the structure of nonnegative equilibria to (3) and their linearized stability. We prove the main result (i.e. Theorem 2) of this paper in section "Global attractivity of a positive equilibrium". Then, in section "Examples and numeric simulations", a suitable example together with its numeric simulations is given to illustrate the feasibility of the main results. We end this paper by a briefly discussion.

\section{Nonnegative equilibria and their linearized stability}

Obviously, (3) has three boundary equilibria, $E_{0}=(0,0), E_{1}=\left(\frac{r_{1}}{b_{1}}, 0\right)$, and $E_{2}=\left(0, \frac{r_{2} k_{2}}{a_{2}}\right)$. Set $B \triangleq a_{1} r_{2}(1-m)^{2}-a_{2} r_{1}(1-m)+a_{2} b_{1} k_{1}$ and $\Delta \triangleq B^{2}-4(1-m)$ $a_{2} b_{1}\left[(1-m) a_{1} r_{2} k_{2}-a_{2} r_{1} k_{1}\right]$. As for the existence of positive equilibria and linearized stability of equilibria, similar to the discussion in Yu (2012), we have the following results:

Case 1. Suppose one of the following conditions holds.

$$
\begin{aligned}
& \text { (i) } m>1-\frac{a_{2} r_{1} k_{1}}{a_{1} r_{2} k_{2}} . \\
& \text { (ii) } m=1-\frac{a_{2} r_{1} k_{1}}{a_{1} r_{2} k_{2}} \quad \text { and } B<0 . \\
& \text { (iii) } m<1-\frac{a_{2} r_{1} k_{1}}{a_{1} r_{2} k_{2}}, \quad B<0, \quad \text { and } \Delta=0 .
\end{aligned}
$$

Then (3) has a unique positive equilibrium $E_{3,1}=\left(x_{3,1}, y_{3,1}\right)$ with $x_{3,1}=\frac{-B+\sqrt{\Delta}}{2(1-m) a_{2} b_{1}}$ and $y_{3,1}=\frac{r_{2}\left((1-m) x_{3,1}+k_{2}\right)}{a_{2}}$.

Case 2. If $m<1-\frac{a_{2} r_{1} k_{1}}{a_{1} r_{2} k_{2}}, B<0$, and $\Delta>0$, then (3) has two positive equilibria $E_{3, \pm}=\left(x_{3, \pm}, y_{3, \pm}\right)$, where $x_{3, \pm}=\frac{-B \pm \sqrt{\Delta}}{2(1-m) a_{2} b_{1}}$ and $y_{3, \pm}=\frac{r_{2}\left((1-m) x_{3, \pm}+k_{2}\right)}{a_{2}}$.

Case 3. If no condition in Case 1 or Case 2 holds, then (3) has no positive equilibrium.

Proposition 1 (i) Both $E_{0}$ and $E_{1}$ are unstable.

(ii) $\quad E_{2}$ is locally asymptotically stable if $m<1-\frac{a_{2} r_{1} k_{1}}{a_{1} r_{2} k_{2}}$ while it is unstable if $m>1-\frac{a_{2} r_{1} k_{1}}{a_{1} r_{2} k_{2}}$.

(iii) The positive equilibrium $E_{3,1}$ in Case $1(\mathrm{i})(\mathrm{ii})$ is stable if $2 b_{1}(1-m)$ $x_{3,1}^{2}-\left(r_{1}(1-m)-r_{2}(1-m)-b_{1} k_{1}\right) x_{3,1}+k_{1} r_{2}>0$.

(iv) The positive equilibrium $E_{3,-}$ is unstable while the positive equilibrium $E_{3,+}=\left(x_{3,+}, y_{3,+}\right)$ is stable if $2 b_{1}(1-m)$ $x_{3,+}^{2}-\left(r_{1}(1-m)-r_{2}(1-m)-b_{1} k_{1}\right) x_{3,+}+k_{1} r_{2}>0$.

When $m=0$ that is there is no prey refuge, Proposition 1 becomes to Propositions 2.1 and 2.2 in $\mathrm{Yu}$ (2012). Thus our results supplement the exist ones. In the coming section, we will prove the main result (i.e. Theorem 2) of this paper.

\section{Global attractivity of a positive equilibrium}

In this section, we first introduce several lemmas which will be useful in proving the main result (i.e. Theorem 2 ) of this paper. 
Lemma 1 (Chen et al. 2007) If $a>0, b>0$ and $\dot{x} \geq x(b-a x)$, when $t \geq 0$ and $x(0)>0$, we have:

$$
\liminf _{t \rightarrow+\infty} x(t) \geq \frac{b}{a} .
$$

If $a>0, b>0$ and $\dot{x} \leq x(b-a x)$, when $t \geq 0$ and $x(0)>0$, we have:

$$
\limsup _{t \rightarrow+\infty} x(t) \leq \frac{b}{a} .
$$

Now, we prove the main result of this paper.

Proof of Theorem 2 Let $(x(t), y(t))^{T}$ be any positive solution of (3). From condition $\left(C_{3}\right)$, we can choose a small enough $\varepsilon>0$ such that

$$
\begin{gathered}
\frac{a_{2} b_{1} r_{1} k_{1}-a_{1}(1-m)^{2} r_{1} r_{2}-a_{1}(1-m) b_{1} r_{2} k_{2}}{a_{2} b_{1} k_{1}} \\
-\left(\frac{a_{1}(1-m)^{2} r_{2}}{a_{2} k_{1}}+\frac{a_{1}(1-m)}{k_{1}}\right) \varepsilon>0 .
\end{gathered}
$$

The first equation of (3) yields

$$
\dot{x}(t) \leq\left(r_{1}-b_{1} x\right) x .
$$

By applying Lemma 1 to (5) leads to

$$
\limsup _{t \rightarrow+\infty} x(t) \leq \frac{r_{1}}{b_{1}} .
$$

Hence, for above $\varepsilon>0$, there exists a $T_{1}>0$ such that

$$
x(t) \leq \frac{r_{1}}{b_{1}}+\varepsilon \triangleq M_{1}^{(1)} .
$$

(6) together with the second equation of (3) leads to

$$
\dot{y}(t) \leq\left(r_{2}-\frac{a_{2} y}{(1-m) M_{1}^{(1)}+k_{2}}\right) y, \quad \text { for all } \quad t \geq T_{1} .
$$

From (7), according to Lemma 1, we can obtain

$$
\limsup _{t \rightarrow+\infty} y(t) \leq \frac{r_{2}\left((1-m) M_{1}^{(1)}+k_{2}\right)}{a_{2}} .
$$

Thus, for above $\varepsilon$, there exists a $T_{2} \geq T_{1}$, such that

$$
y(t) \leq \frac{r_{2}\left((1-m) M_{1}^{(1)}+k_{2}\right)}{a_{2}}+\varepsilon \triangleq M_{2}^{(1)}, \quad \text { for all } t \geq T_{2} .
$$


(8) together with the first equation of (3) leads to

$$
\dot{x}(t) \geq\left(r_{1}-b_{1} x-\frac{a_{1}(1-m) M_{2}^{(1)}}{k_{1}}\right) x, \quad \text { for all } t \geq T_{2} .
$$

According to (4), we can obtain

$$
\begin{aligned}
r_{1}-\frac{a_{1}(1-m) M_{2}^{(1)}}{k_{1}}= & r_{1}-\frac{a_{1}(1-m) r_{2}}{a_{2} k_{1}}\left(\frac{(1-m) r_{1}}{b_{1}}+k_{2}\right) \\
& -\frac{a_{1}(1-m)}{k_{1}}\left(\frac{r_{2}(1-m)}{a_{2}}+1\right) \varepsilon \\
= & \frac{a_{2} b_{1} r_{1} k_{1}-a_{1}(1-m)^{2} r_{1} r_{2}-a_{1}(1-m) b_{1} r_{2} k_{2}}{a_{2} b_{1} k_{1}} \\
& -\left(\frac{a_{1}(1-m)^{2} r_{2}}{a_{2} k_{1}}+\frac{a_{1}(1-m)}{k_{1}}\right) \varepsilon>0,
\end{aligned}
$$

Therefore, by Lemma 1 and (9), we have

$$
\liminf _{t \rightarrow+\infty} x(t) \geq \frac{r_{1}-\frac{a_{1}(1-m) M_{2}^{(1)}}{k_{1}}}{b_{1}} .
$$

Hence, for above $\varepsilon$, there exists a $T_{3} \geq T_{2}$, such that

$$
x(t) \geq \frac{r_{1}-\frac{a_{1}(1-m) M_{2}^{(1)}}{k_{1}}}{b_{1}}-\varepsilon \triangleq m_{1}^{(1)}, \quad \text { for all } t \geq T_{3} .
$$

From (11) and the second equation of system (3), we know that for $t \geq T_{3}$,

$$
\dot{y}(t) \geq\left(r_{2}-\frac{a_{2} y}{(1-m) m_{1}^{(1)}+k_{2}}\right) y .
$$

Applying Lemma 1 to (12) leads to

$$
\liminf _{t \rightarrow+\infty} y(t) \geq \frac{r_{2}\left((1-m) m_{1}^{(1)}+k_{2}^{l}\right)}{a_{2}} .
$$

That is, for above $\varepsilon$, there exists a $T_{4}>T_{3}$ such that

$$
y(t) \geq \frac{r_{2}\left((1-m) m_{1}^{(1)}+k_{2}\right)}{a_{2}}-\varepsilon \triangleq m_{2}^{(1)}, \quad \text { for all } t \geq T_{4} .
$$

From (6), (8), (11) and (13), for $t \geq T_{4}$, we have

$$
0<m_{1}^{(1)} \leq x(t) \leq M_{1}^{(1)}, \quad 0<m_{2}^{(1)} \leq y(t) \leq M_{2}^{(1)} .
$$


(14) together with the first equation of (3) leads to

$$
\dot{x}(t) \leq\left(r_{1}-b_{1} x-\frac{a_{1}(1-m) m_{2}^{(1)}}{(1-m) M_{1}^{(1)}+k_{1}}\right) x, \quad \text { for all } t \geq T_{4} .
$$

From (10) and (14), we have

$$
r_{1}-\frac{a_{1}(1-m) m_{2}^{(1)}}{(1-m) M_{1}^{(1)}+k_{1}}>r_{1}-\frac{a_{1}(1-m) m_{2}^{(1)}}{k_{1}} \geq r_{1}-\frac{a_{1}(1-m) M_{2}^{(1)}}{k_{1}}>0 .
$$

Therefore, similarly to the analysis of (5-6), for above $\varepsilon$, there exists a $T_{5}>T_{4}$ such that

$$
x(t) \leq \frac{r_{1}-\frac{a_{1}(1-m) m_{2}^{(1)}}{(1-m) M_{1}^{(1)}+k_{1}}}{b_{1}}+\frac{\varepsilon}{2} \triangleq M_{1}^{(2)} .
$$

Compare (6) with (15), one can get

$$
M_{1}^{(2)}<M_{1}^{(1)}
$$

Substituting (15) into the second equation of system (3), we have

$$
\dot{y}(t) \leq\left(r_{2}-\frac{a_{2} y}{(1-m) M_{1}^{(2)}+k_{2}}\right) y, \quad \text { for all } t \geq T_{5} .
$$

Applying Lemma 1 to the above inequality leads to

$$
\limsup _{t \rightarrow+\infty} y(t) \leq \frac{r_{2}\left((1-m) M_{1}^{(2)}+k_{2}\right)}{a_{2}}
$$

Thus, for above $\varepsilon$, there exists a $T_{6} \geq T_{5}$, such that

$$
y(t) \leq \frac{r_{2}\left((1-m) M_{1}^{(2)}+k_{2}\right)}{a_{2}}+\frac{\varepsilon}{2} \triangleq M_{2}^{(2)}, \quad \text { for all } t \geq T_{6}
$$

From (8), (16) and (18), we have

$$
M_{2}^{(2)}<M_{2}^{(1)}
$$

Substituting (11) and (18) into the first equation of system (3), we obtain

$$
\dot{x}(t) \geq\left(r_{1}-b_{1} x-\frac{a_{1}(1-m) M_{2}^{(2)}}{(1-m) m_{1}^{(1)}+k_{1}}\right) x, \quad \text { for all } t \geq T_{6} .
$$


According to (10) and (19), we have

$$
r_{1}-\frac{a_{1}(1-m) M_{2}^{(2)}}{(1-m) m_{1}^{(1)}+k_{1}}>r_{1}-\frac{a_{1}(1-m) M_{2}^{(1)}}{k_{1}}>0
$$

Thus, similarly to the above analysis, for above $\varepsilon$, there exists a $T_{7} \geq T_{6}$, such that

$$
x(t) \geq \frac{r_{1}-\frac{a_{1}(1-m) M_{2}^{(2)}}{(1-m) m_{1}^{(1)}+k_{1}}}{b_{1}}-\frac{\varepsilon}{2} \triangleq m_{1}^{(2)}, \quad \text { for all } t \geq T_{7} .
$$

From (11), (19) and (20), we have

$$
m_{1}^{(1)}<m_{1}^{(2)}
$$

It follows from (20) and the second equation of system (3) that

$$
\dot{y}(t) \geq\left(r_{2}-\frac{a_{2} y}{(1-m) m_{1}^{(2)}+k_{2}}\right) y, \quad \text { for all } t \geq T_{7}
$$

Thus, similarly to the above analysis, for above $\varepsilon$, there exists a $T_{8} \geq T_{7}$, such that

$$
y(t) \geq \frac{r_{2}\left((1-m) m_{1}^{(2)}+k_{2}\right)}{a_{2}}-\frac{\varepsilon}{2} \triangleq m_{2}^{(2)}, \quad \text { for all } t \geq T_{8} .
$$

From (13), (21) and (23) , we have

$$
m_{2}^{(1)}<m_{2}^{(2)}
$$

Therefore, it follows from (14), (16), (19), (21) and (24) that

$$
\begin{aligned}
& 0<m_{1}^{(1)}<m_{1}^{(2)} \leq x(t)<M_{1}^{(2)} \leq M_{1}^{(1)}, \\
& 0<m_{2}^{(1)}<m_{2}^{(2)} \leq y(t) \leq M_{2}^{(2)}<M_{2}^{(1)}, \quad \text { for all } t \geq T_{8} .
\end{aligned}
$$

Repeating the above procedure, we get four sequences $M_{i}^{(n)}, m_{i}^{(n)}, i=1,2, n=1,2, \ldots$, such that

$$
\begin{aligned}
& M_{1}^{(n)}=\frac{r_{1}-\frac{a_{1}(1-m) m_{2}^{(n-1)}}{(1-m) M_{1}^{(n-1)}+k_{1}}}{b_{1}}+\frac{\varepsilon}{n}, \quad M_{2}^{(n)}=\frac{r_{2}\left((1-m) M_{1}^{(n)}+k_{2}\right)}{a_{2}}+\frac{\varepsilon}{n} \\
& m_{1}^{(n)}=\frac{r_{1}-\frac{a_{1}(1-m) M_{2}^{(n)}}{(1-m) m_{1}^{(n-1)}+k_{1}}}{b_{1}}-\frac{\varepsilon}{n}, \quad m_{2}^{(n)}=\frac{r_{2}\left((1-m) m_{1}^{(n)}+k_{2}\right)}{a_{2}}-\frac{\varepsilon}{n}
\end{aligned}
$$

Now, We go to show that the sequences $M_{i}^{(n)}$ are non-increasing, and the sequences $m_{i}^{(n)}$, are non-decreasing for $i=1,2$ by induction. Firstly, from (25), we immediately get

$$
M_{i}^{(2)}<M_{i}^{(1)}, m_{i}^{(2)}>m_{i}^{(1)}, \quad i=1,2
$$


Let us suppose that for $\mathrm{n}$,

$$
M_{i}^{(n)}<M_{i}^{(n-1)}, m_{i}^{(n)}>m_{i}^{(n-1)}, \quad i=1,2 .
$$

By direct computation, one can obtain

$$
\begin{aligned}
M_{1}^{(n+1)} & =\frac{r_{1}-\frac{a_{1}(1-m) m_{2}^{(n)}}{(1-m) M_{1}^{(n)}+k_{1}}}{b_{1}}+\frac{\varepsilon}{n+1}<\frac{r_{1}-\frac{a_{1}(1-m) m_{2}^{(n-1)}}{(1-m) M_{1}^{(n-1)}+k_{1}}}{b_{1}}+\frac{\varepsilon}{n}=M_{1}^{(n)} \\
M_{2}^{(n+1)} & =\frac{r_{2}\left((1-m) M_{1}^{(n+1)}+k_{2}\right)}{a_{2}}+\frac{\varepsilon}{n+1}<\frac{r_{2}\left((1-m) M_{1}^{(n)}+k_{2}\right)}{a_{2}}+\frac{\varepsilon}{n}=M_{2}^{(n)} \\
m_{1}^{(n+1)} & =\frac{r_{1}-\frac{a_{1}(1-m) M_{2}^{(n+1)}}{(1-m) m_{1}^{(n)}+k_{1}}}{b_{1}}-\frac{\varepsilon}{n+1}<\frac{r_{1}-\frac{a_{1}(1-m) M_{2}^{(n)}}{(1-m) m_{1}^{(n-1)}+k_{1}}}{b_{1}}-\frac{\varepsilon}{n}=m_{1}^{(n)} \\
m_{2}^{(n+1)} & =\frac{r_{2}\left((1-m) m_{1}^{(n+1)}+k_{2}\right)}{a_{2}}-\frac{\varepsilon}{n+1}<\frac{r_{2}\left((1-m) m_{1}^{(n)}+k_{2}\right)}{a_{2}}-\frac{\varepsilon}{n}=m_{2}^{(n)}
\end{aligned}
$$

Therefore, we have that

$$
\begin{aligned}
& 0<m_{1}^{(1)}<m_{1}^{(2)}<\cdots<m_{1}^{(n)}<x(t)<M_{1}^{(n)}<\cdots<M_{1}^{(2)}<M_{1}^{(1)}, \\
& 0<m_{2}^{(1)}<m_{2}^{(2)}<\cdots<m_{2}^{(n)}<y(t)<M_{2}^{(n)}<\cdots<M_{2}^{(2)}<M_{2}^{(1)},
\end{aligned}
$$

Hence, the limits of $M_{i}^{(n)}$ and $m_{i}^{(n)}, i=1,2, n=1,2, \ldots$ exist. Denote that

$$
\lim _{n \rightarrow+\infty} M_{1}^{(n)}=\bar{x}, \lim _{n \rightarrow+\infty} m_{1}^{(n)}=\underline{x}, \lim _{n \rightarrow+\infty} M_{2}^{(n)}=\bar{y}, \lim _{n \rightarrow+\infty} m_{2}^{(n)}=\underline{y} .
$$

Hence $\bar{x} \geq \underline{x}, \bar{y} \geq \underline{y}$. Letting $n \rightarrow+\infty$ in (26), we immediately

$$
\begin{array}{ll}
r_{1}-b_{1} \bar{x}-\frac{a_{1}(1-m) \underline{y}}{(1-m) \bar{x}+k_{1}}=0, & r_{2}-\frac{a_{2} \bar{y}}{(1-m) \bar{x}+k_{2}}=0 \\
r_{1}-b_{1} \underline{x}-\frac{a_{1}(1-m) \bar{y}}{(1-m) \underline{x}+k_{1}}=0, & r_{2}-\frac{a_{2} \underline{y}}{(1-m) \underline{x}+k_{2}}=0
\end{array}
$$

It follows from (28) that

$$
\begin{aligned}
& a_{2}\left(r_{1}-b_{1} \bar{x}\right)\left((1-m) \bar{x}+k_{1}\right)=a_{1} r_{2}(1-m)\left((1-m) \underline{x}+k_{2}\right), \\
& a_{2}\left(r_{1}-b_{1} \underline{x}\right)\left((1-m) \underline{x}+k_{1}\right)=a_{1} r_{2}(1-m)\left((1-m) \bar{x}+k_{2}\right) .
\end{aligned}
$$

Multiplying the second equality of (29) by -1 and adding it to the first equality of (29), we have

$$
(\bar{x}-\underline{x})\left(a_{1} r_{2}(1-m)^{2}+a_{2} r_{1}(1-m)-a_{2} b_{1} k_{1}-a_{2} b_{1}(1-m)(\bar{x}+\underline{x})\right)=0 .
$$

We claim $\bar{x}=\underline{x}$. Otherwise, $\bar{x} \neq \underline{x}$ and

$$
a_{2} b_{1}(1-m)(\bar{x}+\underline{x})=a_{1} r_{2}(1-m)^{2}+a_{2} r_{1}(1-m)-a_{2} b_{1} k_{1}
$$


Substituting (30) into (29), we have

$$
\begin{aligned}
a_{2}^{2} b_{1}\left(r_{1}-b_{1} \bar{x}\right)\left((1-m) \bar{x}+k_{1}\right)= & a_{1} r_{2}(1-m)\left(a_{1} r_{2}(1-m)^{2}+a_{2} r_{1}(1-m)\right. \\
& \left.-a_{2} b_{1} k_{1}+a_{2} b_{1} k_{2}-a_{2} b_{1}(1-m) \bar{x}\right), \\
a_{2}^{2} b_{1}\left(r_{1}-b_{1} \underline{x}\right)\left((1-m) \underline{x}+k_{1}\right)= & a_{1} r_{2}(1-m)\left(a_{1} r_{2}(1-m)^{2}+a_{2} r_{1}(1-m)\right. \\
& \left.-a_{2} b_{1} k_{1}+a_{2} b_{1} k_{2}-a_{2} b_{1}(1-m) \underline{x}\right) .
\end{aligned}
$$

Thus, $\bar{x}$ and $\underline{x}$ are two positive solutions of the following equation

$$
\begin{aligned}
a_{2}^{2} b_{1}\left(r_{1}-b_{1} x\right)\left((1-m) x+k_{1}\right)= & a_{1} r_{2}(1-m)\left(a_{1} r_{2}(1-m)^{2}+a_{2} r_{1}(1-m)\right. \\
& \left.-a_{2} b_{1} k_{1}+a_{2} b_{1} k_{2}-a_{2} b_{1}(1-m) x\right) .
\end{aligned}
$$

Simplifying (31), one can get

$$
a_{2}^{2} b_{1}^{2}(1-m) x^{2}+a_{2} b_{1}\left(a_{2} b_{1} k_{1}-a_{2} r_{1}(1-m)-a_{1} r_{2}(1-m)^{2}\right) x+D=0,
$$

where $D=a_{2}\left(a_{1}(1-m)^{2} r_{1} r_{2}+a_{1}(1-m) b_{1} r_{2} k_{2}-a_{2} b_{1} r_{1} k_{1}\right)+a_{1} r_{2}(1-m)\left(a_{1} r_{2}(1-m)^{2}\right.$ $\left.-a_{2} b_{1} k_{1}\right)$. (H1) shows that $a_{1}(1-m)^{2} r_{1} r_{2}+a_{1}(1-m) b_{1} r_{2} k_{2}-a_{2} b_{1} r_{1} k_{1}<0$ and $a_{1} r_{2}(1-m)^{2}-a_{2} b_{1} k_{1}<0$. Hence, $D<0$, that is, Eq. (31) does not have two positive solutions. So $\bar{x}=\underline{x}$ and consequently, $\bar{y}=y$. Obviously, $C_{3}$ implies $a_{1}(1-m) r_{2} k_{2}<a_{2} r_{1} k_{1}$ or $m>1-\frac{a_{2} r_{1} k_{1}}{a_{1} r_{2} k_{2}}$, that is, condition (i) of Case 1 holds. Thus (3) has a unique positive equilibrium $\left(x^{*}, y^{*}\right)$ and $\left(x^{*}, y^{*}\right)$ also satisfies (28). Therefor $\bar{x}=\underline{x}=x^{*}$ and $\bar{y}=y=y^{*}$, that is to say

$$
\lim _{t \rightarrow \infty} x(t)=x^{*}, \quad \lim _{t \rightarrow \infty} y(t)=y^{*}
$$

and this completes the proof.

\section{Examples and numeric simulations}

Consider the following example:

$$
\begin{aligned}
& \dot{x}=\left(11-5 x-\frac{2.4 y}{0.6 x+6.5}\right) x, \\
& \dot{y}=\left(8-\frac{2 y}{0.6 x+2}\right) y .
\end{aligned}
$$

In this case, we have $r_{1}=11, b_{1}=5, a_{1}=4, m=0.4, k_{1}=6.5, r_{2}=8, a_{2}=2, k_{2}=2$ and $\quad B=a_{1} r_{2}(1-m)^{2}-a_{2} r_{1}(1-m)+a_{2} b_{1} k_{1}=63.32, \Delta=B^{2}-4(1-m) a_{2} b_{1}[(1-m)$ $\left.a_{1} r_{2} k_{2}-a_{2} r_{1} k_{1}\right]=6519.8$, so

$$
x^{*}=\frac{-B+\sqrt{\Delta}}{2(1-m) a_{2} b_{1}} \approx 1.4521, \quad y^{*}=\frac{r_{2}\left((1-m) x^{*}+k_{2}\right)}{a_{2}} \approx 11.485 .
$$

By simple computation, we also have

$$
a_{1}(1-m)^{2} r_{1} r_{2}+a_{1}(1-m) b_{1} r_{2} k_{2}-a_{2} b_{1} r_{1} k_{1}=-396.28<0 .
$$

Thus, conditions in Theorem 2 are satisfied, hence, system (33) has a unique positive equilibrium $E^{*}=\left(x^{*}, y^{*}\right)$ which is globally attractive. Numerical simulation also confirms our result (see Fig. 1). 


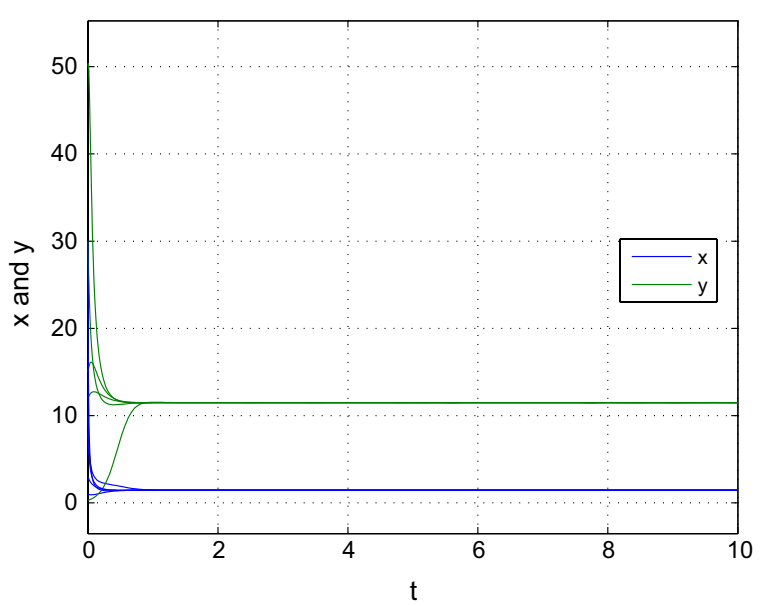

Fig. 1 Dynamic behavior of the system (33) with the initial condition $(x(0), y(0))=(3,12)^{T},(1,30)^{T},(10,0.3)^{T}$, $(8,15)^{T}$ and $(30,50)^{T}$, respectively

\section{Conclusion}

In this paper, we consider a modified Leslie-Gower predator-prey model with Holling-type II schemes and a prey refuge. The structure of equilibria and their linearized stability is investigated. Morever, by using the iterative technique and further precise analysis, sufficient conditions on the global attractivity of a positive equilibrium are obtained. When $m=0$ that is there is no prey refuge, (3) we discussed reduces to (2) which was studied by $\mathrm{Yu}$ (2012). Yu (2012) have provided a sufficient condition on the global asymptotic stability of a positive equilibrium by employing the Fluctuation Lemma and obtained Theorem 1. By comparing Theorems 1 with Corollary 2, we find that the condition $C_{2}$ in Theorem 1 is redundant. Thus our results not only supplement but also improve some existing ones. The numerical simulation of system (33) verify our main results. It follows from Theorem 2 and condition $C_{3}$ that increasing the amount of refuge can ensure the coexistence and attractivity of the two species more easily. This is rational, since the existence of alternate prey can prevent the predator from extinction and increasing the amount of refuge could protect more prey from predation and become permanent. Note that for the diffusion/PDE model where refuge can be spatial, whether refuge can change global attractivity of the interior equilibrium? This is a further problem, which can be studied in the future.

\section{Acknowlegements}

The author would like to thank the anonymous referees and the editor for their constructive suggestions on improving the presentation of the paper. Also, this research was supported by Anhui Province College Excellent Young Talents Support Plan Key Projects (No. gxyqZD2016240).

Competing interests

The author declare that he has no competing interests.

Received: 28 January 2016 Accepted: 31 March 2016

Published online: 14 April 2016 


\section{References}

Aziz-Alaoui MA, Daher OM (2003) Boundedness and global stability for a predator-prey model with modified LeslieGower and Holling-type II schemes. Appl Math Lett 16(7):1069-1075

Chen F, Li Z, Huang Y (2007) Note on the permanence of a competitive system with infinite delay and feedback controls. Nonlinear Anal Real World Appl 8(2):680-687

Chen F, Chen L, Xie X (2009) On a Leslie-Gower predator-prey model incorporating a prey refuge. Nonlinear Anal Real World Appl 10(5):2905-2908

Chen L, Chen F, Chen L (2010) Qualitative analysis of a predator-prey model with Holling type II functional response incorporating a constant prey refuge. Nonlinear Anal Real World Appl 11(1):246-252

Chen F, Wu Y, Ma Z (2012) Stability property for the predator-free equilibrium point of predator-prey systems with a class of functional response and prey refuges. Discrete Dyn Nat Soc 2012. Article ID 148942

Gakkhar S, Singh B (2006) Dynamics of modified Leslie-Gower-type prey-predator model with seasonally varying parameters. Chaos Solitons Fractals 27(5):1239-1255

González-Olivares E, Ramos-Jiliberto R (2003) Dynamic consequences of prey refuges in a simple model system: more prey, fewer predators and enhanced stability. Ecol Model 166(1-2):135-146

Guo H, Song X (2008) An impulsive predator-prey system with modified Leslie-Gower and Holling type II schemes. Chaos Solitons Fractals 36(5):1320-1331

Gupta R, Chandra P (2013) Bifurcation analysis of modified Leslie-Gower predator-prey model with Michaelis-Menten type prey harvesting. J Math Anal Appl 398(1):278-295

Huang Y, Chen Y, Li Z (2006) Stability analysis of a prey-predator model with Holling type III response function incorporating a prey refuge. Appl Math Comput 182(1):672-683

Huo H, Wang X, Chavez C (2011) Dynamics of a stage-structured Leslie-Gower predator-prey model. Math Probl Eng 2011 (2011). Article ID 149341

$\mathrm{Ji}$ C, Jiang D, Shi N (2009) Analysis of a predator-prey model with modified Leslie-Gower and Holling-type II schemes with stochastic perturbation. J Math Anal Appl 359(2):482-498

$\mathrm{Ji}$ C, Jiang D, Shi N (2011) A note on a predator-prey model with modified Leslie-Gower and Holling-type II schemes with stochastic perturbation. J Math Anal Appl 377(1):435-440

$\mathrm{Ji}$ L, Wu C (2010) Qualitative analysis of a predator-prey model with constant-rate prey harvesting incorporating a constant prey refuge. Nonlinear Anal Real World Appl 11(4):2285-2295

Kar TK (2005) Stability analysis of a prey-predator model incorporating a prey refuge. Commun Nonlinear Sci Numer Simul 10(6):681-691

Kar TK (2006) Modelling and analysis of a harvested prey-predator system incorporating a prey refuge. J Comput Appl Math 185(1):19-33

Kar TK, Ghorai A (2011) Dynamic behaviour of a delayed predator-prey model with harvesting. Appl Math Comput 217(22):9085-9104

Ko W, Ryu K (2006) Qualitative analysis of a predator-prey model with Holling type II functional response incorporating a prey refuge. J Differ Eq. 231(2):534-550

Leslie PH (1948) Some further notes on the use of matrices in population mathematics. Biometrika 35(3-4):213-245

Leslie PH (1958) A stochastic model for studying the properties of certain biological systems by numerical methods. Biometrika 45(1-2):16-31

Li Z, Han M, Chen F (2012) Global stability of a stage-structured predator-prey model with modified Leslie-Gower and Holling-type II schemes. Int J Biomath 5:1250057

Liu M, Wang K (2013) Dynamics of a Leslie-Gower Holling-type II predator-prey system with Lévy jumps. Nonlinear Anal 85:204-213

Liu C, Zhang Q, Huang J (2013) Stability analysis of a harvested prey-predator model with stage structure and maturation delay. Math Probl Eng 2013. doi:10.1155/2013/329592. Article ID 329592

Ma Z, Li W, Zhao Y, Wang W, Zhang H, Li Z (2009) Effects of prey refuges on a predator-prey model with a class of functional responses: the role of refuges. Math Biosci 218(2):73-79

Nindjin AF, Aziz-Alaoui MA, Cadivel M (2006) Analysis of a predator-prey model with modified Leslie-Gower and Hollingtype II schemes with time delay. Nonlinear Anal Real World Appl 7(5):1104-1118

Nindjin AF, Aziz-Alaoui MA (2008) Persistence and global stability in a delayed Leslie-Gower type three species food chain. J Math Anal Appl 340(1):340-357

Song X, Li Y (2008) Dynamic behaviors of the periodic predator-prey model with modified Leslie-Gower Holling-type II schemes and impulsive effect. Nonlinear Anal Real World Appl 9(1):64-79

Srinivasu P. Gayatri I (2005) Influence of prey reserve capacity on predator-prey dynamics. Ecol Model 181(2-3):191-202

Tao Y, Wang X, Song X (2011) Effect of prey refuge on a harvested predator-prey model with generalized functional response. Commun Nonlinear Sci Numer Simul 16(2):1052-1059

Yafia R, Adnani F, Talibi Alaoui H (2007) Stability of limit cycle in a predator-prey model with modified Leslie-Gower and Holling-type II schemes with time delay. Appl Math Sci 1(3):119-131

Yafia R, Adnani F, Talibi Alaoui H (2008) Limit cycle and numerical similations for small and large delays in a predator-prey model with modified Leslie-Gower and Holling-type II schemes. Nonlinear Anal Real World Appl 9(5):2055-2067

Yu SB (2012) Global asymptotic stability of a predator-prey model with modified Leslie-Gower and Holling-type II schemes. Discrete Dyn Nat Soc 2012. Article ID 208167

Yu S (2014) Global stability of a modified Leslie-Gower model with Beddington-DeAngelis functional response. Adv Differ Eq. 2014(2014):84

Yu S, Chen F (2014) Almost periodic solution of a modified Leslie-Gower predator-prey model with Holling-type II schemes and mutual interference. Int J Biomath 7(3). Article ID 1450028

Yue Q (2015) Permanence for a modified Leslie-Gower predator-prey model with Beddington-DeAngelis functional response and feedback controls. Adv Differ Eq. 2015:81

Zhu Y, Wang K (2011) Existence and global attractivity of positive periodic solutions for a predator-prey model with modified Leslie-Gower Holling-type II schemes. J Math Anal Appl 384(2):400-408 